\title{
898 INTRATUMORAL ADMINISTRATION OF NL-201, AN ALPHA-INDEPENDENT IL-2/15 RECEPTOR AGONIST, INHIBITS THE GROWTH OF BOTH INJECTED AND UNINJECTED TUMORS IN PRECLINICAL MODELS
}

Laurie Tatalick, Kevin Yu, Justin Huard*, Justin Huard, Marianne Riley, Ryan Swanson, Carl Walkey. Neoleukin Therapeutics, Inc., Redmond, WA, USA

Background NL-201 is a potent, selective, and long-acting computationally designed alpha-independent agonist of the IL2 and IL-15 receptors that is being developed as an immunotherapy for cancer. Intravenous NL-201 administration is active in numerous pre-clinical tumor models. Here, we report data demonstrating favorable tolerability, pharmacokinetics, and antitumor activity of NL-201 after intratumoral (IT) administration in syngeneic murine tumor models.

Methods Mice were implanted with syngeneic colorectal tumors in a single tumor (right flank) or bilateral tumor model (right and left flank). Once tumors were established, mice were randomized to receive IT or intravenous (IV) NL201, or IV vehicle as control. Tumor volumes $\left(\mathrm{mm}^{3}\right)$ and bodyweights were measured twice weekly, and mice were monitored for abnormal clinical signs. Blood samples were collected at pre-specified time points, and serum was analyzed for NL-201 concentration using a ligand-binding assay. Toxicokinetic parameters were determined using a non-compartmental model consistent with the IT and IV routes. Animals were sacrificed if tumor sizes reached $2000 \mathrm{~mm}^{3}$ or if body weight loss was $>20 \%$. In the bilateral tumor models, surviving tumor-free animals from both the IT and IV treatment arms were rechallenged with tumor cells after a washout period to assess antitumor immune responses.

Results IT NL-201 demonstrated dose-dependent antitumor activity and was well tolerated based on a lack of clinical observations and body weight changes at doses up to $10 \mu \mathrm{g} /$ mouse. Comparable antitumor activity was observed in mice receiving $3 \mu \mathrm{g} /$ mouse NL-201 via IV or IT routes, but reduced systemic exposure and better tolerability were observed after IT administration. The estimated absolute bioavailability following $3 \mu \mathrm{g} /$ mouse IT administration was $19.4 \%-66.5 \%$ of the NL-201 IV exposure. In the bilateral tumor model, IT NL-201 resulted in significant antitumor activity in both the treated and untreated tumors. Surviving animals from both IT and IV groups in which the initial tumors regressed rejected engraftment of the same tumor cell line upon rechallenge.

Conclusions IT NL-201 administration resulted in dosedependent antitumor activity in both injected and uninjected tumors while demonstrating markedly reduced systemic exposure and improved tolerability compared to systemic administration at equivalent dose levels. Rechallenged animals failed to develop tumors, demonstrating durable tumor-specific immunity after IT NL-201 treatment. Results of this study support planned clinical investigation of IT NL-201 administration to increase NL-201 concentrations in accessible lesions. Ethics Approval This study was approved by the Institutional Animal Care and Use Committee (IACUC) of CrownBio.

http://dx.doi.org/10.1136/jitc-2021-SITC2021.898 